\title{
HAND GESTURE BASED NAVIGATION CONTROL FOR AUTOMATED WHEELCHAIR
}

\author{
Rajesh K. Nasare ${ }^{1}$ and Ganesh K. Yenurkar ${ }^{2}$
}

\begin{abstract}
The proposed work describes complete design strategy of automated wheelchair which plays more and more important role in modern society for disable people to become locomotive. Nowadays, Human Computer Interface is one of the top research areas and the same is used in the proposed prototype model of automated wheelchair. In this paper image processing concept in MATLAB is used with hardware interface for generation of control signals by using serial communication port. According to the generated control signal the wheelchair either moves left, right, stop, and up.

Keywords: Automated wheelchair, Image Processing, MATLAB, gesture recognition, Communication port.
\end{abstract}

\section{INTRODUCTION}

Gestures are expressive meaningful body motions - i.e. the movements of any part of the body such as fingers, hands, arms, head, face, or body itself with the intent to convey some required information or interact with the environment. So many aspects of gestures are there that may be relevant and therefore may need to be represented explicitly. It can be categorized as follows:

- $\quad$ Spatial information - Where it occurs, locations a gesture refers to.

- $\quad$ Pathic information - The path that a gesture takes.

- $\quad$ Symbolic information - The sign that a gesture makes.

- $\quad$ Affective information - The emotional quality of a gesture.

The number of senior citizens and handicapped people are increasing and accordingly the need of human friendly wheelchair is also consistently increased. Traditional power wheelchairs are having buttons or joysticks for controlling the wheelchair but some disable people are so affected that they can't even apply that much force because of psychomotor problems. Human interface of intelligent wheelchair for easy operation is the most popular research area. Yi Zang, Jiao Zang [1], proposed an intelligent wheelchair system based on hand gesture recognition again but they were used neural network concept and trained the system, In this article that factor by using classification techniques has been removed and real time approach is used.

\footnotetext{
${ }^{1}$ Rajiv Gandhi College Of Engineering and Research, Nagpur

${ }^{2}$ Yeshwantrao Chavan College OF Engineering, Nagpur
} 


\section{RELATED WORK}

Many more such systems have been implemented like Speech recognition or Voice recognition [3], Motion detection [4], Head gesture recognition [5], Hand gesture recognition using ADA Boost Algorithm [15], Oral Motion detection='n [6], and Forehead bio signals recognition [7]. But each system has certain drawbacks which are reflected in table no 1.

\section{SYSTEM DESIGN AND METHODOLOGY}

The stereo matching method is divided into the area-based method and the feature-based method. Although the area-based method can be divided into several methods, the SAD method is implemented with a depth map by calculating the matching region in a lot of computational stereo vision images in real-time. In the SAD matching region except the left and right nonmatching region, our system makes the depth map using the left and right image displacement difference. This needs calibration images as input. Thus, this is added calibration. Even though this corrects lens distortion, this system has intrinsic camera distortion. Also the left and right cameras have tiny differences in hardware. For this reason, although it is applied to the SAD method, the displacement difference isn't 0 .

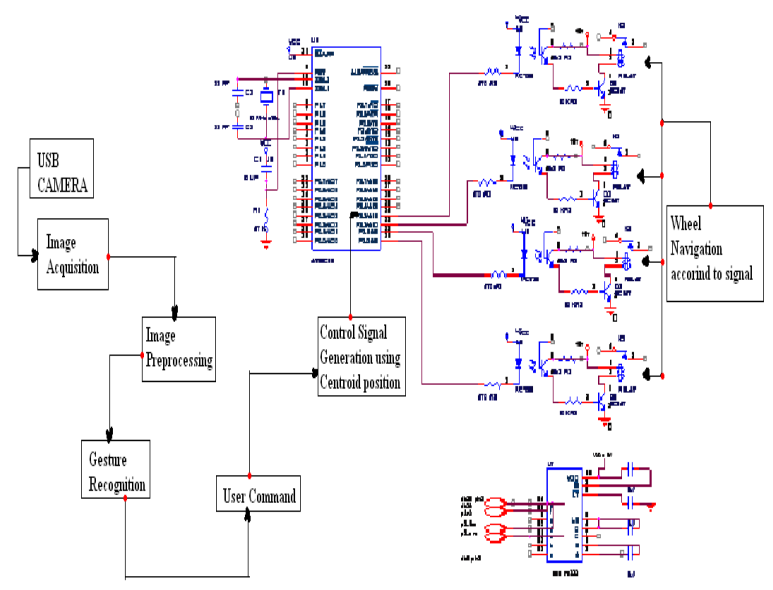

Fig. System Architecture

Equation (1) calculates that the smallest displacement difference is the matched displacement difference.

$$
O(x, u, \delta)=\sum_{n=0}^{w h-1} \sum_{k=0}^{W W-1} \mid I_{R}(x, u)-\left(I_{\delta}(x-\delta, u) \mid-\cdots(1)\right.
$$

wh $=$ window height

$\mathrm{wW}=$ window width

$\delta=$ differential values

$I_{R}(x, u)=$ intensity corresponding to coordinate of the left image. 
$I_{L}(x, u)=$ intensity corresponding to coordinate of the right image.

For computing the matching points of two images, it is calculated that the displacement difference is changed from 0 to 1 . RIP the optimal matching point is selected as the smallest among several values. The optimal displacement difference isn't correctly calculated as 0 , due to the internal and external camera distortion. The SAD method is selected due to its high-precision and the advantage of the real-time guarantee. Using the displacement difference of the left and right images and the focal distance, we find the 3 -dimensional coordinates in each frame.

$d=b \times f / d_{1}-d_{r} \times$ width

width $=640$ pixels

$\mathrm{b}($ base-line $)=12 \mathrm{~cm}$

$\mathrm{f}($ focal-length $)=0.38 \mathrm{~cm}$

$\mathrm{d}$ (distance)

we can't calculate all pixel distances. Because of the occlusion by the foreground object we can determine the approximate distance information using pixel interpolation.

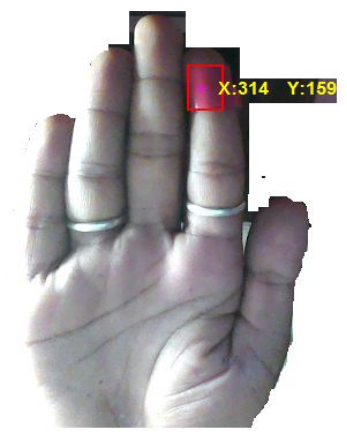

Fig. pixel interpolation

We present the depth map with color representing distance in order of red, orange, yellow, green and blue. We recognize the foreground object as a hand. However we can't perform postprocessing operations using only the detected result because of the non-matching region. Based on the acquired hand detection image, we obtain the center coordinate of the hand. This point is used to make a circle and the method is used to remove the background except the circle. However, because the background is not removed completely, we finally detect the hand using YCBCR, skin detection. Because the hand region is not normalized, the hand size is reduced or increased. For normalizing the image of the hand, we find the outline around the hand. Based on this outline, we make a rectangular grid with the image of the hand. We make the 110110 image using warping with each vertex of the rectangle. For diminishing the influence of lighting, we use the gray image. Changes in the gray image are represented by the difference of light and shade. For equalization of the difference of light and shade, we use histogram equalization. And then we can acquire the hand image of light and shade smoothing.

\section{IMPLEMENTATION}


Hand gestures receive the most attention among those who study gesture - in fact, many (if not most) references to gesture recognition only consider hand gestures. The vast majority of automatic recognition systems are for deictic gestures (pointing), emblematic gestures (isolated signs) and sign languages (with a limited vocabulary and syntax). Some are components of bimodal systems, integrated with speech recognition. Some produce precise hand and arm configuration while others only coarse motion. Hand gestures provide a huge diversity in signal generation for controlling the navigation of various directions. System design has been implemented through following steps:
A) Camera Interfacing
B) Blob Analysis and Centroid creation
C) Image Conversion
D) Noise Removal
E) Control Signal Generation

\section{A. Camera Interfacing}

1. $\quad$ Select Start > Programs > CMU 1394 Camera > 1394 Camera Name.

2. The application opens a window, shown in the following figure.

3. From the Camera Demo application, select Camera > Check Link. This option causes the demo application to look for DCAM-compatible cameras that are available through the IEEE 1394 (FireWire) connection. The demo application displays the results of this search in a pop-up message box. In the following example, the demo application found a camera. Click OK to continue.

4. Select Camera > Select Camera and select the camera you want to use. The Select Camera option is not enabled until after the Check Link option has successfully found cameras. 5. Select Camera > Init Camera. In this step, the demo application checks the values of various camera properties. The demo application might resize itself to fit the video format of the specified camera. If you see the following dialog box message, click yes.

6. Select Camera > Show Camera to start acquiring video.

7. To exit, select Stop Camera from the Camera menu and then click Exit.

\section{B. Blob analysis and Centroid creation}

1. There are several motivations for studying and developing blob detectors. One main reason is to provide complementary information about regions, which is not obtained from edge detectors or corner detectors. In early work in the area, blob detection was used to obtain regions of interest for further processing. These regions could signal the presence of objects or parts of objects in the image domain with application to object recognition and/or object tracking. In other domains, such as histogram analysis, blob descriptors can also be used for peak detection with application to segmentation. Another common use of blob descriptors is as main primitives for texture analysis and texture recognition. In more recent work, blob descriptors have found increasingly popular use as interest points for wide baseline stereo matching and to signal the presence of informative image features for appearance-based object recognition based on local image statistics. There is also the related notion of ridge detection to signal the presence of elongated objects. 
Connectivity checks are carried out by checking the labels of pixels that are North-East, North, North-West and West of the current pixel (assuming 8-connectivity). 4-connectivity uses only North and West neighbors of the current pixel. The following conditions are checked to determine the value of the label to be assigned to the current pixel (4-connectivity is assumed)

Conditions to check:

1. Does the pixel to the left (West) have the same value as current pixel?

1. Yes - We are in the same region. Assign the same label to the current pixel

2. $\quad$ No - Check next condition

2. Do both pixels to the North and West of the current pixel have the same value as current pixel but not the same label?

1. Yes - We know that the North and West pixels belong to the same region and must be merged. Assign the current pixel the minimum of the North and West labels, and record their equivalence relationship

2. No - Check next condition

3. Does the pixel to the left (West) have a different value and the one to the North the same value as current pixel?

1. Yes - Assign the label of the North pixel to the current pixel

2. No - Check next condition

4. Do the pixel's North and West neighbors have different pixel values than current pixel?

1. Yes - Create a new label id and assign it to the current pixel

The label equivalence relationships generated are,

Table 2 Label Relationships

\begin{tabular}{|l|l|l|}
\hline $\begin{array}{l}\text { Sr. } \\
\text { No. }\end{array}$ & $\begin{array}{l}\text { Set } \\
\text { ID }\end{array}$ & Equivalent Labels \\
\hline 1 & 1 & 1,2 \\
\hline 2 & 2 & 1,2 \\
\hline 3 & 3 & $3,4,5,6,7$ \\
\hline 4 & 4 & $3,4,5,6,7$ \\
\hline 5 & 5 & $3,4,5,6,7$ \\
\hline 6 & 6 & $3,4,5,6,7$ \\
\hline 7 & 7 & $3,4,5,6,7$ \\
\hline
\end{tabular}

The next figure illustrates how the block calculates the perimeter of a blob when you set the Connectivity parameter to 8 . 


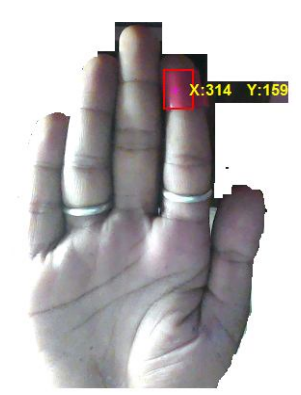

Fig. Centroid and bounding created on hand

\section{Image conversion}

Converting images from one class to another is a common operation. When converting between classes, keep in mind the value ranges of the classes being converted. A double-precision array, $\mathrm{B}$, is generated by the command $\mathrm{B}=$ double (A). If $\mathrm{C}$ is an array of class double in which all values are in the range $[0,255]$ (but possibly containing fractional values), it can be converted to an uint 8 array with the command $\mathrm{D}=$ uint $8(\mathrm{C})$. If an array of class double has any values outside the range $[0,255]$ and it is converted to class uint8 in the manner just described, MATLAB converts IS to 0 all values that are less than 0 , and converts to 255 all values that are greater than 255. Numbers in between are rounded to the nearest integer. Thus, proper scaling of a double array so that its elements are in the range $[0,255]$ is necessary before converting it to uint 8 . As indicated in Section 2.6.2, converting any of the numeric data classes to logical creates an array with logical $1 \mathrm{~s}$ in locations where the input array has nonzero values and logical 0s in places where the input array contains 0s.

The Median Filter block replaces the central value of an $M-b y-N$ neighborhood with its median value. If the neighborhood has a center element, the block places the median value there, as illustrated in the following figure. The block has a bias toward the upper-left corner when the neighborhood does not have an exact center. See the median value placement in the following figure.

\section{Noise removal}

The block pads the edge of the input image, which sometimes causes the pixels within [M/2 N/2] of the edges to appear distorted. The median value is less sensitive than the mean to extreme values. As a result, the Median Filter block can remove salt-and-pepper noise from an image without significantly reducing the sharpness of the image.

If the data type of the input signal is floating point, the output has the same data type. The data types of the signals input to the "I" and "Val" ports must be the same.

\section{E. Control signal generation}

For wheelchair navigation control the signal are required to send to DC Motor and according to the value which has been programmed, the wheelchair navigates.

\section{E.1 Centroid Bounding}


If PROPERTIES is the string 'all', REGIONPROPS returns all of the shape measurements. If called with a grayscale image, REGIONPROPS also returns Pixel value measurements. If PROPERTIES is not specified or if it is the string 'basic', these measurements are computed: 'Area', 'Centroid', and 'BoundingBox'.

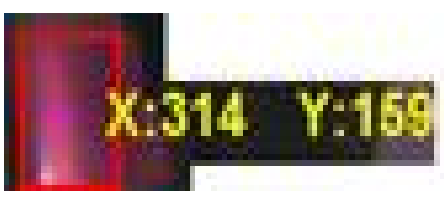

Fig. Centroid of Rectangular Box

\section{E.2 Screen region partition}

Now whatever region is available, it is divided into four areas and based on the pixel value of position the required control signals are generated.

\begin{tabular}{|c|c|}
\hline$X<=250 \& \& Y>=250$ & $X>=250 \& \& Y>=250$ \\
TOP & STOP \\
\hline$X<=250$ \&\& $Y<=250$ & $X>=250 \& \& Y<=250$ \\
LEFT & RIGHT \\
\hline
\end{tabular}

Fig. Screen Region Partition

\section{RESULTS}

The results are taken by randomly generating the control signals based on hand gesture movement and CL via microcontroller it is passed to the DC Motor. Whatever value is passed like 00, 01, 10, 11 the wheel chair moves either stop, left, right, and top. The results are shown as follows:
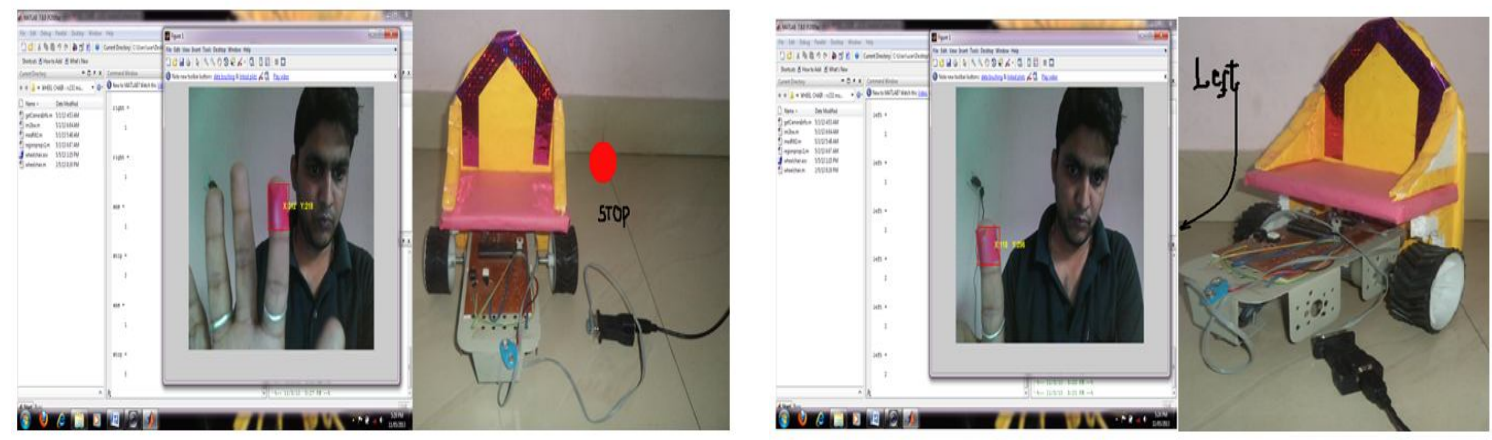
Fig. Signals to STOP

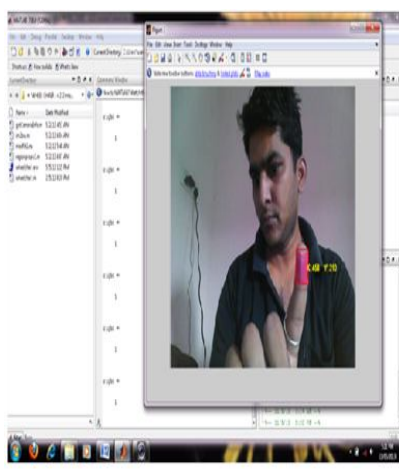

Fig. Signals to RIGHT
Fig. Signals to LEFT
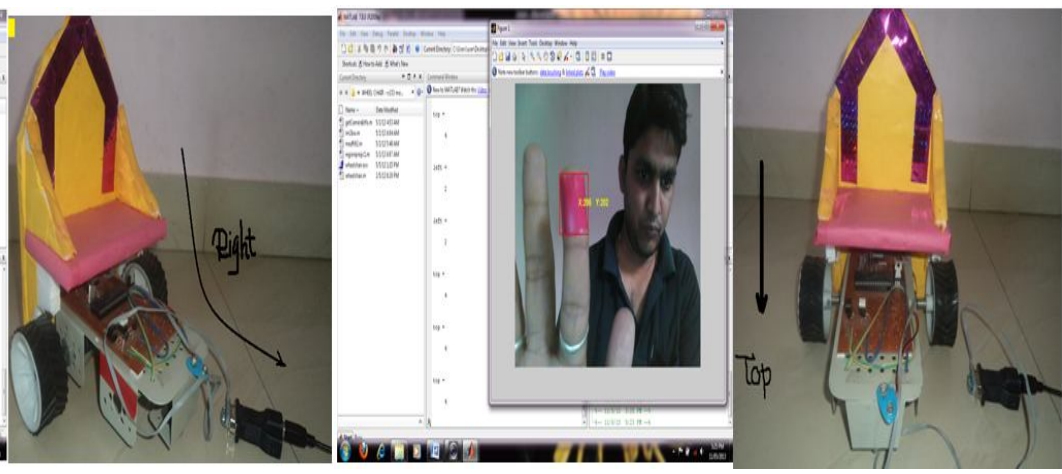

Fig. Signals to TOP

Graph clearly indicates that number of vehicles with respect to new approach is much less as compared to old approach. The congestion on the signal is comparatively reduced.

\section{Result Analysis}

Result analysis for the taken sample has been shown as follows:

\begin{tabular}{|l|l|l|l|}
\hline $\begin{array}{l}\text { Control } \\
\text { Signal }\end{array}$ & $\begin{array}{l}\text { Number of } \\
\text { Samples }\end{array}$ & $\begin{array}{l}\text { Error } \\
\text { Count }\end{array}$ & $\begin{array}{l}\text { Recognition } \\
\text { Quality }\end{array}$ \\
\hline Left & 42 & 0 & $100 \%$ \\
\hline Right & 40 & 0 & $100 \%$ \\
\hline Stop & 45 & 6 & $86.67 \%$ \\
\hline Top & 52 & 4 & $92.30 \%$ \\
\hline
\end{tabular}

Table 4 Results of signal recognition

- Rate of Momentum: $10 \mathrm{~cm} / \mathrm{s}$

- Response time: Real Time

- $\quad$ Accuracy Rate: $96.23 \%$

- Delay Time: approx Nil

- Frame Acquisition: 1 Frame/5ms

\section{CONCLUSION}

Hand gesture recognition based man-machine interface is being developed vigorously in recent years. Gesture recognition is also important for developing alternative human computer interaction modalities. It enables human to interface with machine in a more natural way. MATLAB provides the better solution for hand gesture recognition. The Canny Edge Detection 
Algorithm for the purpose of detecting points at which image brightness changes sharply or formally. We used ANN algorithm for gesture identification. ANN has a fast computational ability. Edge detection and ANN provides good and powerful solution for hand gesture recognition using MATLAB. A novel intelligent wheelchair system controlled by hand gesture was proposed. Haar-like features and AdaBoost algorithm was used for hand gesture detection. The results of detection combined with centroid are used to determine control commands. The experiments show that the system is robust. However, the hand gesture is a little simple. In future work, more classifiers will be trained to recognize different hand posture for comfortable controlling. This system remains to be further improved.

\section{FUTURE SCOPE}

As a near future, we can generate more control signals by using more bit values. Here in this approach we have generated only four control signals i.e. for left, right, top, and stop. Hand Gesture Recognition system is very useful for the physically impaired persons. The system can be trained to help these people to communicate with each other. In this system we have only considered the static gesture, but in real time we need to extract the gesture form the video or moving scene. Therefore the system needs to be upgraded to support dynamic gesture. This system can be further upgraded to give order and control robots. It can also be very helpful for the physically impaired persons. All the above methods can be further enhanced for binary and color images. Some more applications are that this Hand Gesture Recognition system can be in case of games. Instead of using the mouse or keyboard, we can use some pre-defined hand gesture to play any game. Also, this system can be used to operate any electronic devices by just keeping a sensor which recognizes the hand gestures. Another application is that this can be used for security and authorization by keeping any particular hand gesture as the password.

We look forward to facilitate rich interactive features which would enable the users to interact and take portability to the next level. Use of smaller packages of the integrated circuits will scale down the size of the device to that of a watch, thereby improving the portability.

\section{REFERENCES}

[1] S. M. Torres Muller," Brain-computer Interface Based on Visual Evoked Potentials to Command Autonomous Robotic Wheelchair" in IEEE Transaction on Medical and Biological Engineering 2010. 30(6) p. 407-416.

[2] R. Barea, M. Mazo, Wheelchair guidance strategies using EOG. Journal of Intelligent and Robotic Systems, 34(3):279-299, 2011.

[3] Akira Murai, Masaharu Mizuguchi, "Voice Activated Wheelchair with Collision Avoidance Using Sensor" in IEEE Conference 2009. P. 4232-4237.

[4] Nobuyuki Sekine, “ Development of Intelligent Power Wheelchair Assisting for People on Daily Life using Motion Recognition" in IEEE Workshop on Advance Motion Control March 2010. P. 302-306.

[5] Zhang-fang Hu Lin Liu, "A Novel Intelligent Wheelchair Control Approach Based On Head Gesture Recognition", in IEEE conference on computer application and System Modeling 2010. P. V6/159-V6/163.

[6] Takeshi Saitoh, and RyosukeKonishi, "OralMotion Controlled Intelligent Wheelchair" International Conference, Essex University, United Kingdom,Proceedings of the IEEE 2008.

[7] Lai Wei and Kui Yuan, "Use of Forehead Bio-signals for controlling an intelligent wheelchair", in IEEE Transaction on Robotics and Biomimetics 2008. P. 108-113.

[8] Paulo Trigueiros," A Comparison of Machine Learning Algorithms Applied to Hand Gesture Recognition", in IEEE Conference 2012. P. 260-267.

[9] Terauki Ito," Preliminary Study On Gesture Recognition For Walking Stick Interface”, in IEEE Conference on Intelligent Systems, Modelling and Simulation 2010. P. 65-69.

[10] Terauki Ito, "Simple Gesture Distinction for Brief Message Exchange", in IEEE Conference on Analytical Modelling and Computer Simulation 2010. P. 18-22. 
[11] Michal Lech, Bozena Kostek," Gesture-based Computer Control System applied to the Interactive Whiteboard", in IEEE Proceeding on Information Technology 2010. P. 75-78.

[12] Rustam Rakhimov Igorevich," Hand gesture recognition algorithm based on grayscale histogram of the image ", in IEEE Conference on Computing Applications 2010. P. 4244-4247.

[13] Shweta. K. Yewale,"Rapid Hand Gesture Recognition Using Different Algorithms Based on Artificial Neural Network", in IEEE Conference 2011. P. 287-292.

[14] Yi Zhang, Jiao Zhang," A Novel Intelligent Wheelchair Control System Based On Hand Gesture Recognition", in IEEE Proceeding on Complex Medical Engineering 2011. P. 334-339.

[15] Faria, B.M., and L.P. Reis. Classification of Facial Expressions Using Data Mining and machine Learning Algorithms. in IEEE conference on Information Technology 2009.

[16] Gillian, N.E., Gesture Recognition for Musician Computer Interaction, in Music Department 2011. P. $206-211$.

[17] Faria, B.M., et al., Machine Learning Algorithms applied to the Classification of Robotic Soccer Formations ans Opponent Teams, in IEEE Conference on Cybernetics and Intelligent Systems (CIS) 2010: Singapore. p. 344 349.

[18] Maldonado-Báscon, S., et al., Road-Sign detection and Recognition Based on Support Vector Machines, in IEEE Transactions on Intelligent Transportation Systems2007. p. 264-278.

[19] Vicen-Bueno, R., et al., Complexity Reduction in Neural Networks Applied to Traffic Sign Recognition Tasks, 2004. 